\title{
ADDENDUM 23.01.2020
}

Medizinprodukte in der präklinischen Notfallmedizin - die Defibrillatorweste

Gaiser J. retten! 2019; 8(05): 310-313; doi:10.1055/a-0658-8808

Zum oben genannten Artikel ergänzt der Autor folgenden Warnhinweis:

Um zu vermeiden, dass Rettungskräfte durch eine Schockabgabe gefährdet werden, darf der Patient nur berührt werden, wenn keine Alarme und keine akustischen Ansagen zu hören sind. Zur eigenen Sicherheit ist das System durch die Entnahme des Akkus zu deaktivieren; dann können Rettungsteams Diagnostik und Reanimationsmaßnahmen mit eigenem Gerät und den aktuellen Reanimationsleitlinien entsprechend weiterführen.

Ein aktives System überwacht den Herzrhythmus des Patienten. Detektiert die Defibrillatorweste einen defibrillierbaren Herzrhythmus (z. B. Kammertachykardie bzw. Kammerflimmern), wird zunächst eine Alarmsequenz (Vibrationsalarm + akustischer Zweiton-Alarm) gestartet, die es dem wachen Patienten ermöglicht, eine Schockabgabe durch Drücken von Reaktionstasten zu verhindern. Ist der Patient bewusstlos, werden bei Kammerflimmern nach ca. 30 Sekunden (je nach ärztlicher Programmierung) Personen in unmittelbarer Umgebung durch den fortgesetzten Sirenenalarm und den zusätzlichen Sprachhinweis „Es besteht die Gefahr eines Elektroschocks - den Patienten nicht berühren! Umstehende Personen dürfen nicht eingreifen!” vor der bevorstehenden Schockabgabe gewarnt . Vor der ersten Schockabgabe kommt es zunächst zum Austreiben von blauem Kontaktgel, danach wird der Schock (ca. 75-150 J) abgegeben, und zwar in einer Sequenz von bis zu 5 Schocks in einem zeitlichen Abstand von ca. 60 Sekunden. Anschließend wird eine neuerliche komplette Ablaufsequenz eingeleitet, und es werden ggf. neuerliche Alarm- und Schocksequenzen abgegeben.

Weiteres Informationsmaterial für Rettungskräfte findet sich unter https://lifevest.zoll.com/de/medical-professionals/first-responderresources.

\section{Jörg Gaiser, Sindelfingen}

